\title{
AERODYNAMICS AND AEROACOUSTICS SURVEY FOR A LOW SPEED SUBSONIC JET OPERATING AT MACH 0.25
}

\begin{abstract}
A. R. Proença $a^{a}$,
O. de Almeida ${ }^{a}$, and R. H. Self ${ }^{\mathrm{b}}$

${ }^{a}$ Universidade Federal de Uberlândia

Faculade de Engenharia Mecânica

Campus Santa Mônica

Uberlândia, Minas Gerais, Brasil andsproenca@gmail.com

${ }^{\mathrm{b}}$ University of Southampton Institute of Sound and Vibr. Research Highfield, Southampton, SO17 1BJ Hampshire, UnitedKingdom.

Received: October 29, 2014

ABSTRACT

The purpose of this work is to study and characterize, in laboratory, the aerodynamics of a free jet operating at subsonic regime and identify its acoustic signature. This study aims to analyze the fundamental role of turbulent flow structures in the total noise produced at different Mach numbers. This work is focused at low speed subsonic jets operating at Mach number 0.25 . The research is done by analyzing the data obtained in experiments using Pitot tube, hot-wire anemometer and acoustic measurements. This work also describes the experimental procedures for each step of analysis, as well as the characteristics of jet noise facility. The data from measurements with Pitot tube is used to study the mean velocity profiles. The average properties are also analyzed with anemometry system, likewise used to study the turbulent intensity of eleven axial lines, ranging from the center line to the edge of the nozzle (lipline). These results are compared with the literature and is verified the accuracy of hot-wire anemometers for turbulent intensities lower than $15 \%$. A database with the sound pressure level as a function of frequency is constructed from experiments serving as data for further numerical analysis to solve this problem.
\end{abstract}

Revised: November 30, 2014

Accepted: December 30, 2014
Keywords: subsonic jets, aeroacoustics, aerodynamics, hot-wire anemometry, pitot tube

\section{NOMENCLATURE}

$\bar{U} \quad$ normalized velocity

$U_{o} \quad$ mean velocity at a specified location, $\mathrm{m} / \mathrm{s}$

$U_{\max }$ maximum velocity at the nozzle exit, $\mathrm{m} / \mathrm{s}$

\section{INTRODUCTION}

The noise produced by an aircraft can be heard as a whole, however it is generated by different parts and by the interaction of external flow and these components. According to the aircraft performance, during each phase of flight, one region or equipment should contribute more or less to the "total noise". In other words, the aircraft on the ground, while taxing, on run-up from the jet exhaust, during the take-off, underneath to departure and arrival paths, over-flying while in route and landing, produces different noise not only in terms of amplitude but also in its composition - Figure 1 .

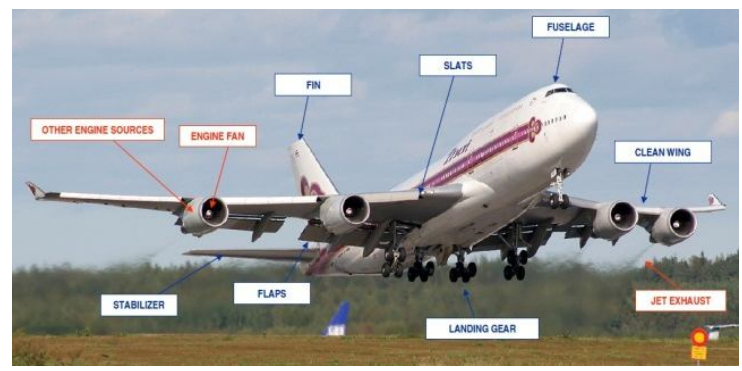

Figure 1. Aircraft and noise sources.
Regarding engine noise specifically, many efforts have been made to understand the noise produced by such sources and its sections separately. The turbines and other mechanical noise are the mainly responsible for the noise produced during the take-off and climb and in a general point of view the major sources of aircraft noise. During the approach the engine noise is also considerable; however, due to the advances in engine technology, the airframe is somewhat noisier during this phase. The engine noise also can be divided into two general categories: internally generated noise or turbo machinery noise and externally generated noise or jet noise. The turbo machinery noise can also be divided from its components, comprised of fan noise, compressor noise, combustion or core noise and turbine noise. Although high bypass-ratio turbofans have considerable fan noise, the jet noise is responsible for the majority of engine noise (Almeida, 2009). This subject is extremely important and is the main motivation of this work.

The aerodynamic noise is also important and is caused by the airflow around the aircraft fuselage and control surfaces. Likewise the first case presented the noise increases as a function of velocity. The air density either is an important factor, which within low altitudes the noise is louder than high altitudes. Also as the engine noise, different aircraft components can produce different sources of noise. Thus, main wings, tail plane, slats, trailing edge flaps, fuselage, landing gear and wheel bays are the most important individual noise producers, in special the 
high-lift devices and the landing gear (Smith, 1989). Figure 2 brings information about the noise during the take-off and approach according to the sources related to the airframe and engine.

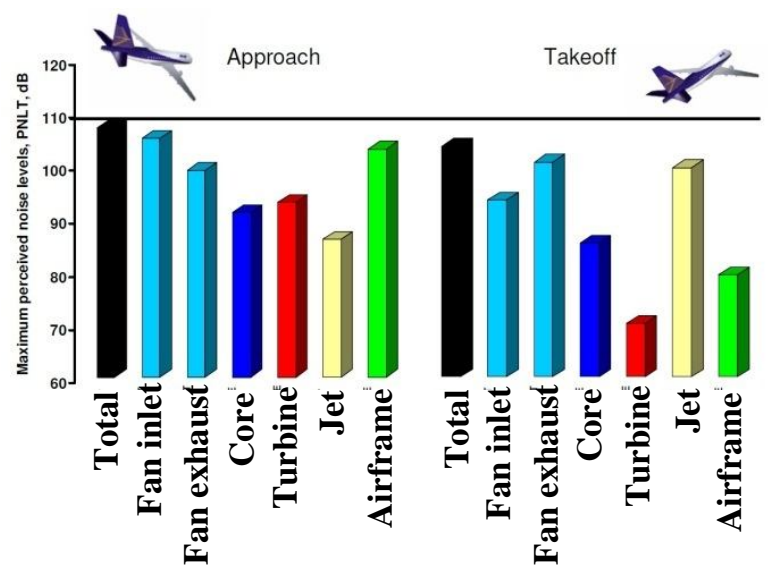

Figure 2. Noise component breakdown at take-off and approach (Almeida, 2009).

The aircraft systems like the cockpit, cabine pressurization conditioning systems, Auxiliary Power Unit (APU) and others also increase the total noise, although its components are not too much expressive as the others stated before.

This work is related to jet study and consequently applied to basic of jet engines or also called gas turbine engines. The jet engines are present in most of passenger and military aircraft nowadays. They can be classified according to type of compressor (centrifugal, axial and centrifugal-axial flow) and also the path the air takes through the engine and how power produced.

The term "jet noise" is usually taken to mean the noise of jet-powered aircraft. However, strictly speaking, it is about only those sources associated with the mixing process between the exhaust flow of the engine and the atmosphere, as was said before during the division in jet and turbo machinery noise. This mixing noise is the only component generated during subsonic operations. In super critical jet there is also a secondary noise source which appears from shock-waves. The present work looks into subsonic regimes.

\section{Ways to Study the Problem}

Probably the most ancient way of investigating a subjectthat we turn our attention herein is to perform controlled experiments. In this case, real scale experiments often become prohibited since the costs involved are too high. In a world where things change so frequently and new products are expected, experiments with such costs are not interesting to manufacturers. Scale experiments or reduced models are very important in this context and the knowledge about the data and its extrapolations are really useful. By considering semi-empirical or empirical methods, it can be said that these methods are fast, reliable and have a long application in industry. However, one of the greatest problems in these methods is the restriction or applied considerations used to derivate them and also the envelope of operating conditions to be applied. As tailored tools for some specific jet noise predictions, many times they are restrictive to certain geometries, velocities, temperature and pressure ranges. Nevertheless, it is also observed that companies and organizations are interested in predicting the noise from jets using methods related to particular cases, and, sometimes, for just only one family of aeronautical engines.

Part of the present work was developed jointly with Embraer S. A., a Brazilian aircraft manufacturer, by means of two projects: "AeronaveSilenciosa: Uma InvestigaçãoemAeroacústica", which was funded by the São Paulo Research Foundation (FAPESP) and EMBRAER S.A and "Desenvolvimento de soluçõesaprimoradas, através de ensaiosaeroacústicos, para o problema de ruídoexterno de aeronaves - DSA", developed by Research Increment and Industrial Improvement Foundation (FIPAI) and EMBRAER S.A., the first and second phases of the EMBRAER's project, respectively.

This work is also part of a cooperation project between the University of Uberlandia (Aeronautical Engineering Course) and the University of Southampton, specifically the Institute of Sound and Vibration Research. The jet aerodynamic and acoustic results were provided in the ISVR's laboratory called DOAK Lab. The test matrix was built up in beginning of August, 2011, and the measurements were made with the supervision of the Fluid Dynamic and Acoustics Group - FDAG. The laboratory in question is an anechoic chamber built to subsonic and supersonic acoustic measurements, this work also was done to extend the applications of the DOAK Lab to aerodynamic measurements, what will be shown in details in sequel.

\section{EXPERIMENTAL METHODOLOGY}

The present work proposes a comprehensive study about the physics of a subsonic jet by aerodynamic and acoustic surveys aiming to better understanding the mechanisms of generation and propagation of noise in aircraft engines.

First of all, it is given a brief description of the Doak Laboratory, the Rolls Royce UTC facility located at ISVR, University of Souhthampton. Information about the flow control and the data acquisition system will be given in the sequence.

The ISVR's Doak laboratory is a $15 \mathrm{~m} \times 7 \times 5 \mathrm{~m}$ anechoic chamber fully anechoic down to $400 \mathrm{~Hz}$. The four walls, ceiling and the floor are covered with wedge type absorbent material. A non-forced exhaust system is composed by a rectangular collector section allowing air to pass through into a small secondary 
acoustic chamber. It contains two silent cold jet rigs of 2" and 4" pipe diameter. The larger of the rigs is located on the centerline of the chamber and is used primarily for the investigation of full-scale bleed valves, having an in-line silencer. On the other hand, the smaller rig utilizes a labyrinth pot silencer and can be moved along the chamber. They are fed from two high pressure compressed air (20 bar) from two storage tanks and the range of velocity available for testing is Mach 0.2 to 1 . Therefore, single jets measurements can be performed on flow regimes characteristic of civil aircraft. Both polar and a transversable azimuthal array of microphones can be used to give a complete three-dimensional sound field. Next figure (Fig. 3) shows a picture of the anechoic chamber when the 4 " rig was being used to bleed valve investigation.

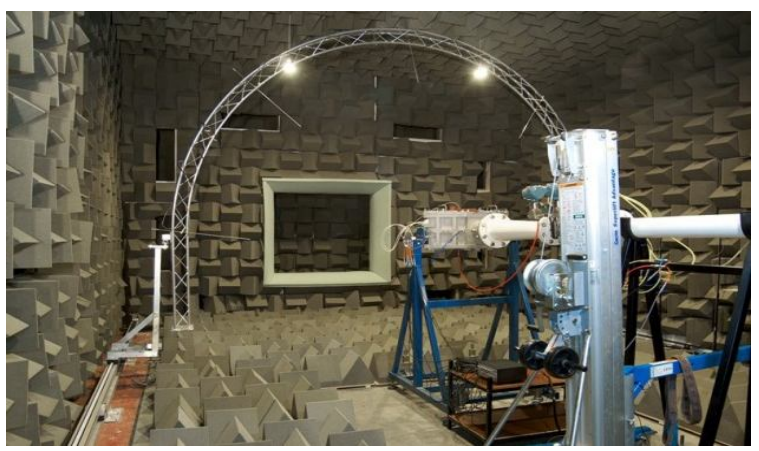

Figure 3. Anechoic chamber at DOAK Laboratory during antransversable azimuthal array of microphones experiment.

There are some nozzle options and the one used in this work was a $38.1 \mathrm{~mm}$ exit-diameter, convergent, conical nozzle used for most of the tests done at the Doak Laboratory. This nozzle was chosen because its aerodynamic and acoustic characteristics were welldocumented in the Noise Test Facility (NTF) at QinetiQ, Farnborough, UK.

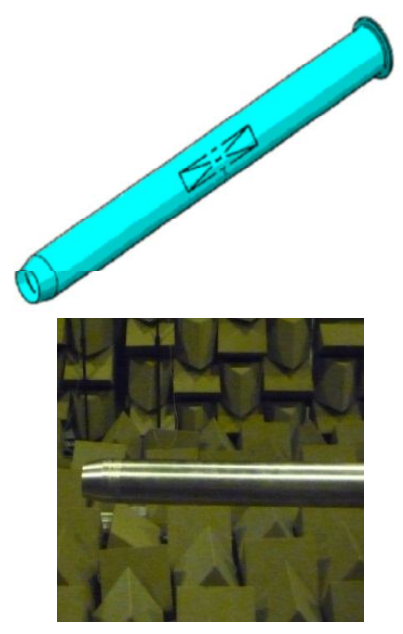

Figure 4. Sketch and picture of $38 \mathrm{~mm}$ reference nozzle.
The present work was carried out with the 2" rig. The labyrinth silencer was placed in the middle of the chamber, just after the larger rig. The cold, subsonic jet was operated from the nozzle showed above. In order to run aerodynamics measurements a traverse system was placed inside the anechoic room. Details of these changes can be found in the next figure and compared to the previous one.

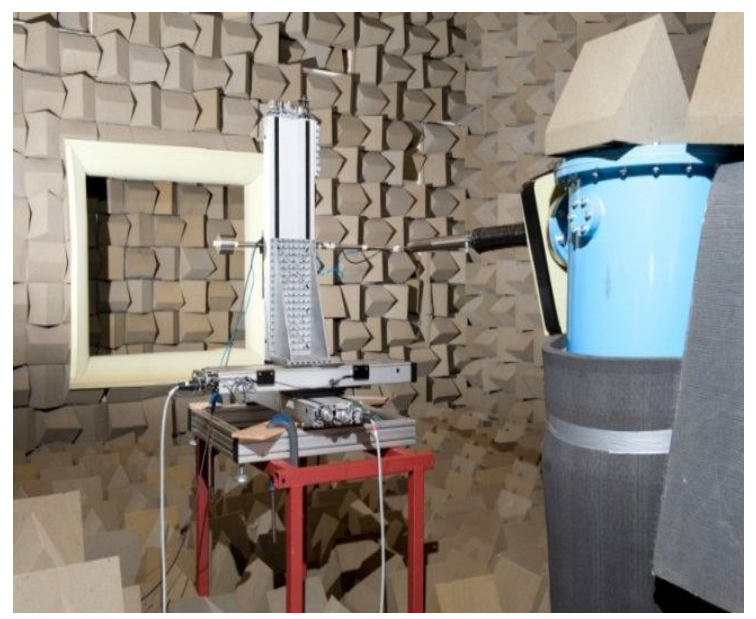

Figure 5. General view of Doak Laboratory with traverse system to hot-wire anemometer and Pitot tube measurements.

Acoustic data is acquired using GRAS Type 40BF microphones and digitized using a National Instruments NI PCI-4472 acquisition card with a $102.4 \mathrm{kHz}$ sample rate, and 24-bit resolution. The time domain data is then transformed to the frequency domain using a 4096 point Hamming window-averaged Fourier transform. All subsequent data processing is done using narrowband spectra. Microphones are calibrated using a B\&K Type 4220 pistonphone calibrator. Measurements of Overall Sound Pressure Level are achieved by numerically integrating the narrowband spectra with respect to frequency using a trapezium rule method across the entire range of narrowband frequencies. The narrowband data may also be transformed into onethird octave band spectra using idealised third-octave filters consistent with ANSI S1.1-1986.

Aerodynamic data is acquired using Druck PDCR 820 pressure sensors and digitised using the National Instruments data acquisition hardware described above. The pressure sensors are calibrated using a Druck Type DPI 610 pressure calibrator.

\section{Aerodynamics Measurements}

The measurements of the mean flow velocity profiles were performed using a Pitot tube, while the hot-wire anemometer is used for mean flow (to compare with Pitot tube) and turbulence intensity measurements. 


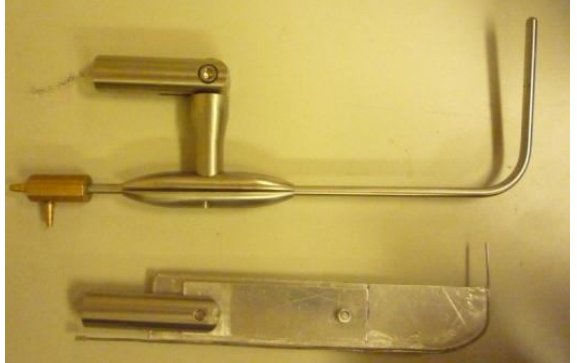

Figure 6. Pitot tube of the micromanometer and Pitot tube built during the experiments by Mat Gruber.

The Pitot tube is used to measure mean flow velocity profiles and the spreading of the jet. Furthermore, it was used as a reference velocity measurement to calibrate the hot-wire sensors. Two different transducer systems, coupled to the Pitot tube, were used in these experiments: a micromanometer for Pitot tube, allowing velocities up to $180 \mathrm{~m} / \mathrm{s}$ and a Druk PDCR 820 transducer. Both data is digitalized using the National Instruments data acquisition board.

Hot-wire anemometry is the main measuring system applied in this work. Single hot-wire anemometers are the most common sensors applied in flow measurements, for several reasons: reduced size, price relatively low, high frequency response, simple to use. One of the limitations is that it has to be used for low turbulence intensities (up to 10\%). Various sensors had been used in this work. Each probe has its specification for resistance and operation resistance given by the manufacturer. Next figure shows one of the single hot-wire anemometer used and the configuration with the temperature probe.

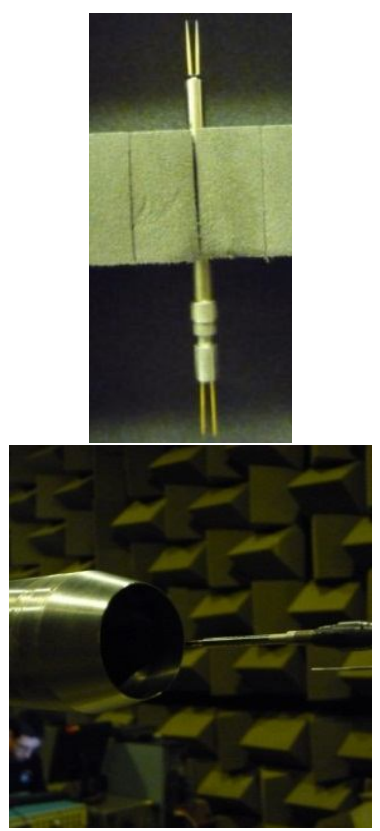

Figure 7. Single hot-wire sensor.
The velocity distributions were acquired along the jet axis to different radial positions and the workspace of mean velocity profile experiments is demonstrated in the Fig. 8. The nozzle centre is situated in '0", vertical axis, ' $y$ ', and represents radial variations in relation the radius of the nozzle and ' $x$ ' is the jet axis. Red points symbolize where the data were acquired. Only for single hot-wire probes, just the points inside the blue rectangle were acquired. Thus, for each Mach number analyzed, 963 points were recorded to Pitot tube and triple hot-film measurements, whilst 583 to single hot-wire.

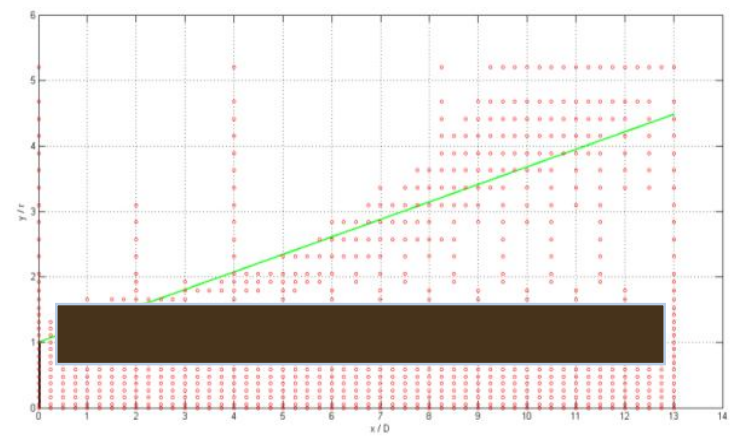

Figure 8. Acquisition points along the region of the jet for aerodynamic measurements.

\section{Acoustics Measurements}

This section shows the equipment used to perform the acoustic measurements. It is mainly composed by microphones, preamplifiers and amplifiers. Far-field noise measurements were acquired using polar microphones covering 6 angles as shown in the Tab. 1 .

Table 1. Far-field microphone location.

\begin{tabular}{|c|c|c|c|}
\hline $\begin{array}{c}\text { Mic. } \\
\text { number }\end{array}$ & $\begin{array}{c}\text { NI } \\
\text { channel }\end{array}$ & $\begin{array}{c}\text { Mic. } \\
\text { angle } \theta\end{array}$ & Mic. type \\
\hline 1 & 3 & $40^{\circ}$ & 1/4" GRAS 40BF \\
\hline 2 & 4 & $50^{\circ}$ & 1/4" GRAS 40BF \\
\hline 3 & 5 & $60^{\circ}$ & 1/4" GRAS 40BF \\
\hline 4 & 6 & $75^{\circ}$ & 1/4" GRAS 40BF \\
\hline 5 & 7 & $90^{\circ}$ & 1/4" GRAS 40BF \\
\hline 6 & 8 & $110^{\circ}$ & 1/4" GRAS 40BF \\
\hline
\end{tabular}

Measurements were done with 1/4" GRAS Type 40BF microphones with a frequency range of $10 \mathrm{~Hz}$ to $100 \mathrm{kHz}$ and dynamic range of $40 \mathrm{~dB}$ to $174 \mathrm{~dB}$ (reference $20 \mu \mathrm{Pa}$ ). This is a precision condenser microphone for general purpose measurements in open acoustic fields and it is externally polarized. As a free-field microphone, it is for measuring the sound pressure which existed before it was placed in the sound field pointing towards the sound source, and its disturbing effects are negligible for most application frequencies due the small dimensions. The normal sensitivity of the microphones used is $4 \mathrm{mV} / \mathrm{Pa}$ and it was checked before each experiment day. 
The acoustic measurements were performed only to the far-field. Six different observer angles [40], [50], [60], [75], [90] e [110] were applied to acquire the noise signature.

\section{EXPERIMENTAL RESULTS}

This sub-section considers the mean velocity profiles acquired by the Pitot tube and the single sensor hot-wire anemometer. The results are disposed in axial velocity profiles, radial velocity profiles and a jet characterization of the whole region measured.

\section{Aerodynamics Results}

The aerodynamic experiments were carried out originally to compose the axial profiles. The next figures present the results for velocity distribution.

Figures 9 to 10 are extracted from Pitot tube measurements. Firstly, Fig. 9 shows the normalized mean velocity from the centerline to lipline at Mach number 0.25 , distributed along the jet axis domain, from 0 to 13 diameters. Each line is normalized independently, according to the Equation:

$$
\bar{U}=\frac{U_{0}}{U_{\max }}
$$

where $U_{0}$ is the velocity measured in meters per second and $U_{\max }$ is the maximum velocity measured in meters per second along that line.

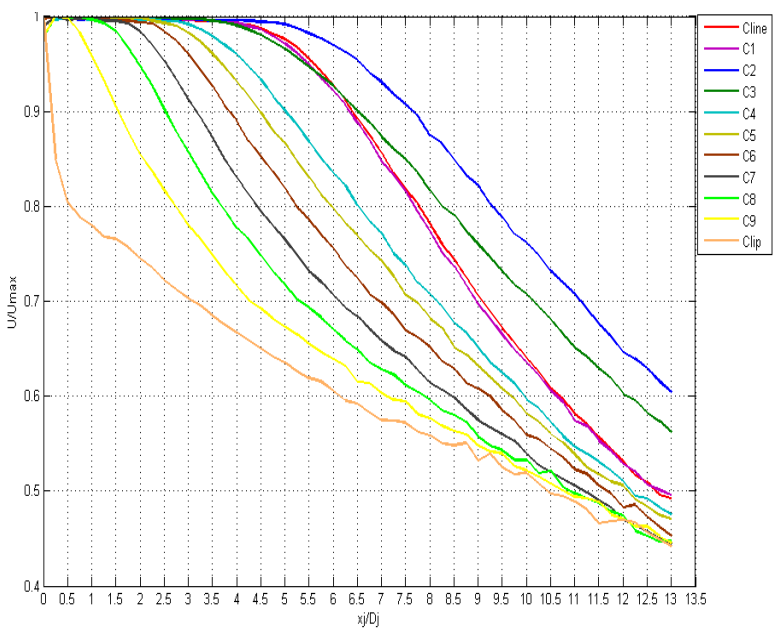

Figure 9. Velocity distribution along the jet axis for different radial positions (Pitot tube, Mach 0.25).

The lines "Cline" and "C1" represents the measurements along the axial line in radial positions 0 and $1.05 \mathrm{~mm}$ from the center of the nozzle. The velocity is expected to be maximum and unchangeable until 4 to 5 diameters from the exit nozzle along the centerline, since it is the potential core length (Abramovich, 1963). According to the Fig. 9, the potential core ends between 4 to 4.5 diameters downstream the jet axis.

The behavior of "C2" and "C3" is unexpected. Since the potential core has a conical shape, the greater the distance from the centerline, the higher velocity decay. It is observed from the other profiles, from "C4" to "Clip". Although all measurements were corrected because the flow variation, the data from these two lines are very discrepant, since it occurred an instantaneous flow velocity increasing, probably related to the rise of pressure in the compressor for an unknown reason.

Keep moving in the radial position over the nozzle region, it can be seen a new trend in the axial profiles. Next figure shows this trend from "S1" to "S20", again for Mach 0.25.

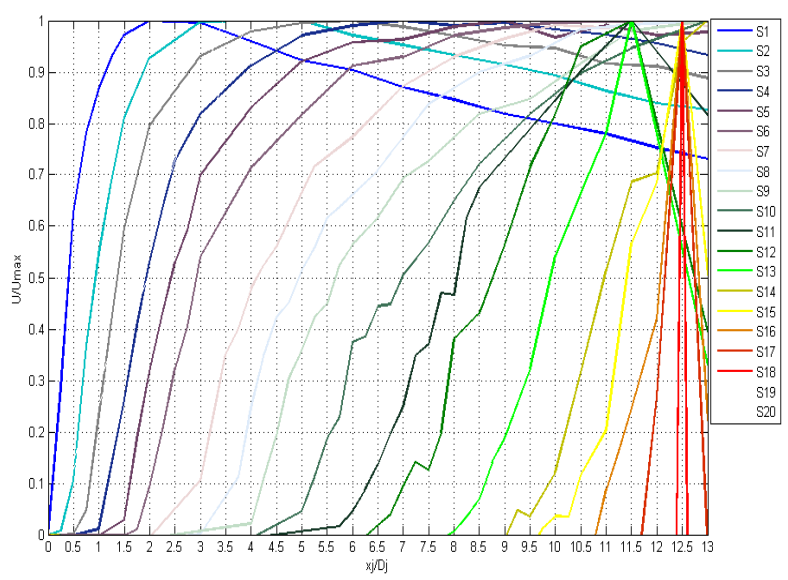

Figure 10. Velocity distribution along the jet axis for different radial positions (Pitot tube, Mach 0.25).

The trend is clearly the expected. In the regions close to the nozzle, there is a sudden increase in the velocity as the sensor moves in the axial position. As the measurements get in lines far from the nozzle, the profile velocity is smaller and takes its maximum velocity at higher axial distances. The trend shows clearly the shear layer between the jet and the stationary flow (ambient), located for each point where the velocity profile reaches the maximum value. The last results (especially after "S17") the jet flow is practically all mixed with the ambient and the value of ' $u$ ' velocity component is close to zero.

Now the same sequence is shown to measurements using a hot-wire sensor. Comparing the quality of the pitot and hot-wire techniques for the measurements discussed, it is clear that the pitot data present curves much smoother than the anemometer. The reason is that the latter is more sensitive to the flow variation. Furthermore, the hotwire calibration curve, a fourth order polynomial, is more complex than the linear curve used by the pressure transducer. As the results had to be corrected to the unsteady pressure imposed by the compressor system, the accumulated errors are most evident in 
the anemometry system.

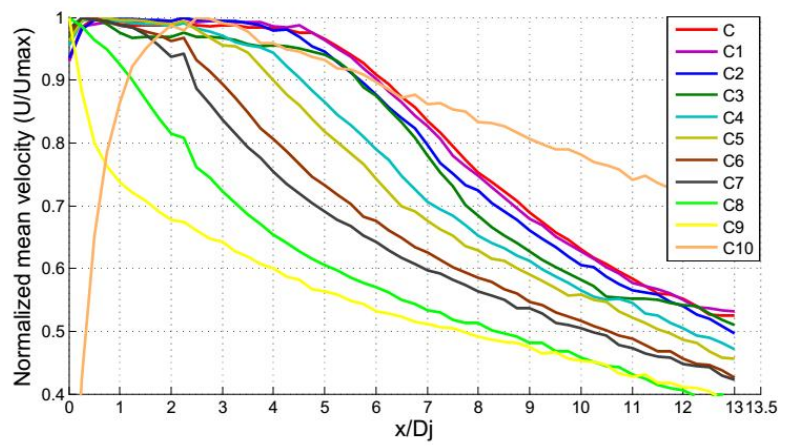

Figure 11. Velocity distribution along the jet axis for different radial positions (Single hot-wire, Mach $0.25)$.

In order to check the trend and the accuracy of the data showed above, the axial profiles obtained with each system are confronted. The centerline for Mach conditions 0.25 is also compared to other experimental references. Figure 12 presents this comparison. It can be seen that at Mach number 0.25 the axial velocity profiles from the pitot and the hotwire system are very similar. Far away around eleven diameters from the exit nozzle, the hot-wire results shown some inconsistency. It is believed that the cause is again the flow variation imposed by the compressor system. The work of Morris and Zaman (2010) shows only the results for Mach 0.25 , alleging the fragility of the modern hot-wires probes with high frequency response to high and unsteady flows. The trend is confirmed, despite a presence of an offset.

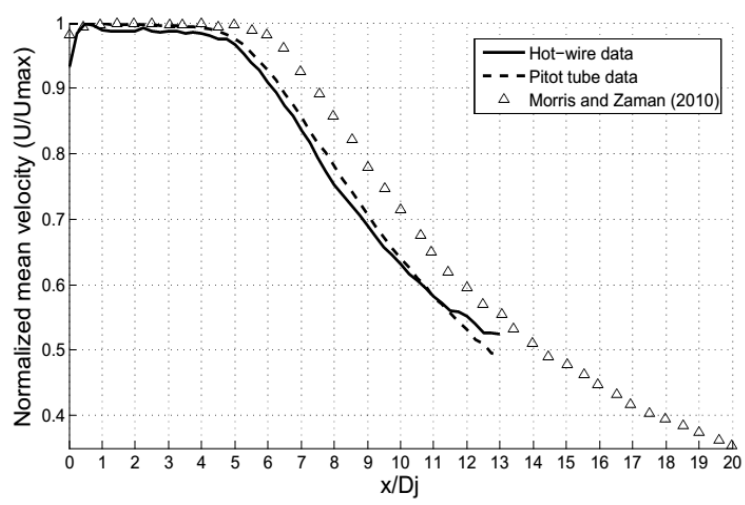

Figure 12. Velocity distribution along the jet axis for centerline at Mach 0.25.__ Hot-wire; _ _ Pitot tube; A Morris and Zaman (2010).

\section{Jet Characterization}

This sub-section is used to present a general view of the jet's velocity distribution. All lines measured are considered and, such as in the previous sub-section, the data is mirrored to better characterize the jet. Although it seems to be merely illustrative, it helps to summarize the information given on the previous sub-sections

Figure 13 shows the velocity distribution from the exit nozzle to thirteen diameters downstream the jet axis, also covering two diameters from the center of the nozzle. It shows the results for the Mach 0.25. These data were acquired by the pitot tube.

It is possible to see the potential core and the shear layer behavior along the imposed velocity regimes. The slight growth of the potential core with the velocity increases is evident, as well as the shear layer spreading, with the small thickness close to the nozzle.

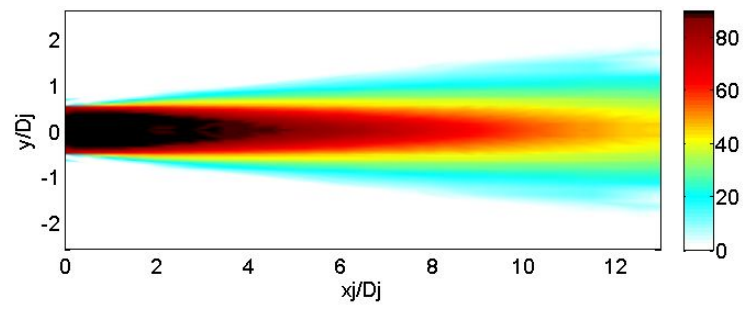

Figure 13. Jet characterization with pitot tube data for Mach 0.25.

\section{Acoustics Results}

The power spectral density - PSD is a way to show the strength of energy variations as a function of frequency. The usual unit of PSD is energy per frequency, and the integral of the PSD over a given frequency range computes the average power in the signal. This spectrum is computed directly by a Fast Fourier Transform method - FFT or by computing an autocorrelation function and transforming it.

In the present work, the Welch's method is used to calculate the PSD of the signal acquired by the anemometer. This method uses a FFT to obtain an estimation of the PSD based on time averaging over short modified periodograms (Welch, 1967).

Welch based on Barllett's method, which consists in splitting the original points into a number "n" of data segments of length "L", compute the periodogram by the discrete Fourier transform, and then compute the squared magnitude of the result dividing by the L. Finally, the result of the periodograms is time-averaged for the $\mathrm{n}$ data segments. The difference is that the on the Welch's method, the system is divided into overlapping segments, which are windowed in the time domain before apply the discrete Fourier transform. It is considered an improvement on the standard periodogram and Bartlett's methods, since it reduces noise in the estimated power spectra when it reduces the frequency resolution.

The processing is done by the Matlab pwelch function, which returns the two-sided Welch PSD estimation. It was used a Hanning window and a sample frequency of $50 \mathrm{kHz}$ (which is transformed in $25 \mathrm{kHz}$ ).

Still taking into account the fact that it is only 
one component velocity, some results to approximate the power spectrum density and compares with the power spectrum of turbulence are performed.

The Fig. 14 shows the result for some points along the "C1", and "C9" lines, for Mach 0.25. The points inside the potential core have less energy than the points in the mixing layer. The low frequencies are responsible by the energy injection, whilst it is seen that the high frequencies are dissipating this energy.

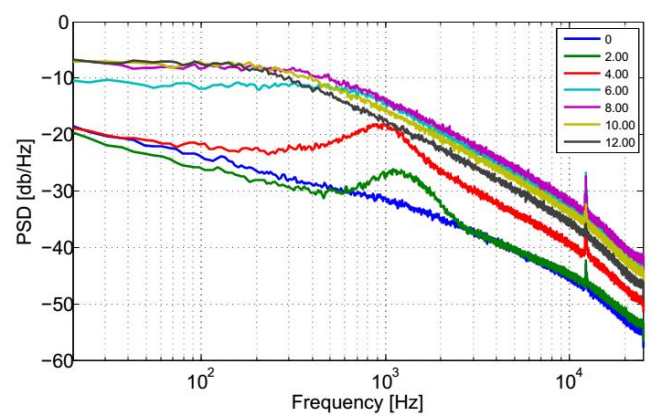

(a)

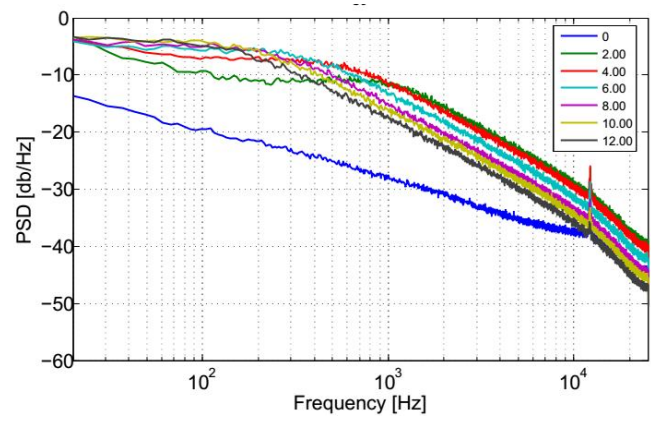

(b)

Figure 14. Power spectral density in measurements at Mach number 0.25.x/Dj=0, 2, 4, 6, 8, 10 and 12. (a) C1; (b) C9.

The SPL pattern is now presented in observer angle comparisons. Due the directivity of the sound and the effects overprint, the sound sources are convected downstream by the mean flow and the maximum noise is radiated at observer angles between 30 and $50^{\circ}$.

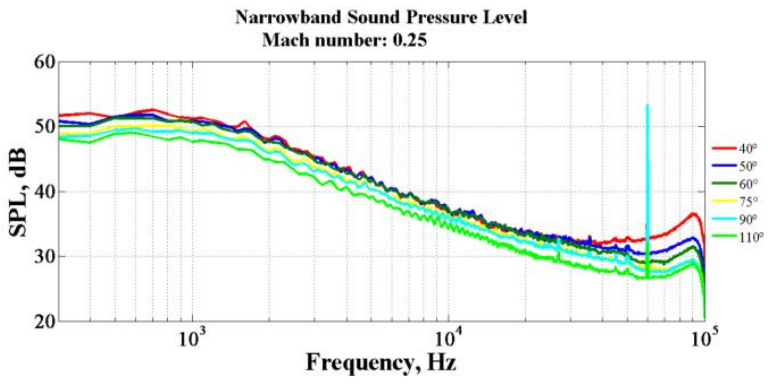

Figure 15. Sound Pressure Level for Mach number 0.25 in six different angles $\left(40^{\circ}, 50^{\circ}, 60^{\circ}, 75^{\circ}, 90^{\circ}\right.$ and $\left.110^{\circ}\right)$.

\section{CONCLUDING REMARKS}

The present work deals with the jet aerodynamics and acoustics through experimental techniques, exploring the basic theory related to low speed subsonic jets. The main goal was to study fundamental of this kind of flow configuration, set up and run measurements from different systems and compare the results with the literature reviewed. It represents a particular achievement to the Aeroacoustics Group recently founded at MFLAB/UFU.

The results are discussed in a methodical order. Firstly, it is presented the aerodynamic work, which compounds most of the efforts in terms of experiments, post-processing and analyses. It shows mean velocity profiles acquired by the pitot tube and hot-wire anemometer sensor. The experiments were carried out to several axial lines, and by combining these points it is also possible estimate the radial profiles, as well as a characterization of the subsonic jets regions. Through the anemometer data, it is also possible analyses the unsteadiness velocity, estimating the turbulence intensity in axial profiles and the power spectral density of the points measured. The profiles are compared with the literature for Mach numbers 0.25 presenting a good agreement.

Secondly, the acoustic results are shown to six observer positions for a wide range of velocities. The data is confronted to the theory related to jets, and it shows a good agreement. The experiments done in this section are extremely simple in the way that many researchers are developing advanced techniques to jet noise measurements, but it is essential to the development of complex sets of experiments and insights about the jet physics, extremely important to the creation of semi-empirical methods. A database is created from these measurements and may apply in future to validate numerical analyses which are underway.

\section{ACKNOWLEDGEMENTS}

The authors would like to thank the financial support provided by Coordenação de Aperfeiçoamento de Pessoal de Ensino Superior (CAPES), Brazil, for the development of this work. They also thanks for all the support received at the Institute of Sound and Vibration for the conclusion of his MsC thesis and the Fundação de Amparo à Pesquisa do Estado de Minas Gerais (FAPEMIG).

\section{REFERENCES}

Almeida, O., 2009, Aeroacoustics of DualStream Jets with Application to Turbofan Engines, Doctoral Thesis, Aeronautics Institute of Technology, São José dos Campos. 
Smith, M. J. T., 1989, Aircraft Noise, Cambridge Aerospace Series.

Abramovich, G. N., 1963, The Theory of Turbulent Jets, The MIT Press Classics.

Morris, P. J., Zaman, and K. B. M. Q., 2010, Velocity Measurements in Jets with Application to Noisesource Modeling, Journal of Sound and Vibration, Vol. 329, pp. 394-414.

Welch, P. D., 1967, The Use of Fast Fourier Transform for the Estimation of Power Spectra: A Method Based on Time Averaging Over Short, Modified Periodograms, IEEE Transactions on Audio Electroacoustics, AU-15, pp. 70-73. 